Optical Society of America. Hitoshi Fujii, Silverio P. Almeida, and James E. Dowling, "Rotational matched

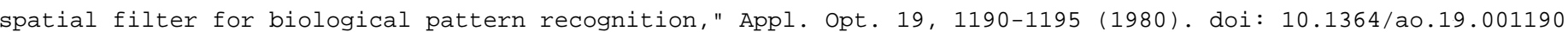

\title{
Rotational matched spatial filter for biological pattern recognition
}

\author{
Hitoshi Fujii, Silverio P. Almeida, and James E. Dowling
}

\begin{abstract}
Randomly oriented microbiological patterns are recognized by rotating a matched spatial filter with an optical wedge prism. The filter is made from a composite input pattern with various sized objects to cover wider ranges of size variation in a species to be identified.
\end{abstract}

\section{Introduction}

One major problem in the practical use of complex matched spatial filters (MSFs) for pattern recognition is known to be extremely high sensitivity to orientation and size variation of input patterns. ${ }^{1,2}$ In particular, the drawbacks are more serious in the recognition of biological species, such as the diatoms treated in our past research, ${ }^{3}$ as shown in Fig. 1 . Diatoms are microscopic water algae found in any body of water such as rivers or ponds. It has been shown ${ }^{4,5}$ that water quality may be measured by the types and numbers of diatoms in bodies of water. Therefore, diatom communities have been studied by many biologists to assess the effects of pollution on water resources. Since it requires time and expertise for proper identification of these organisms with traditional taxonomic means, considerable effort has been made to develop an efficient and rapid biomonitoring system using MSFs. ${ }^{6-8}$ The difficulties in this process are shown in the photograph taken through a microscope. One can easily recognize twelve diatoms in this photomicrographic picture that can be immediately classified into seven different species. However, the standard technique of MSFs has been inadequate for this kind of pattern. ${ }^{9}$ For instance, it is difficult to make a filter that correlates only to the four diatoms at the center of the picture because of their $50 \%$ size variation and random orientations. One so-

When this work was completed, the authors were at Virginia Polytechnic Institute \& State University, Physics Department, Blacksburg, Virginia, 24061; H. Fujii is now with Hokkaido University, Department of Engineering Science, Faculty of Engineering, Sapporo, Hokkaido, Japan and S. P. Almeida is on leave at Universidade Federal de Pernambuco, Departamento de Fisica, 50.000 Recife, Brazil.

Received 29 October 1979.

0003-6935/80/071190-06\$00.50/0.

(C) 1980 Optical Society of America. lution to this problem is to simplify a given input pattern to reduce its sensitivity to rotation and size variation and make a filter, exposed multiple times, for these simplified patterns in several different orientations. ${ }^{8}$ It may also be possible to rotate the input pattern to be analyzed with precisely made image rotation devices. ${ }^{2,10}$ However, those devices are usually very expensive and require difficult alignment procedures.

In this paper, a simpler method is developed to recognize these microbiological patterns by rotating the MSF with an optical wedge prism. The filter is made from various sized diatoms so that it can be used to cover wider ranges of size variation. ${ }^{11}$ The idea of rotational filtering was discussed in a paper by Vander Lugt, ${ }^{12}$ but no one seems to have used it in a practical application.

\section{Experimental Arrangement}

The experimental setup is shown in Fig. 2. An input pattern was placed in a liquid gate, at the back focal plane of the Fourier transform lens $L_{1}$, and a Vander Lugt type $\mathrm{MSF}^{12}$ was made at its focal plane with a reference beam. Then the input pattern was replaced by a new input pattern, such as in Fig. 1, to be analyzed. The developed holographic plate $H$ is set at its original plane with an optical wedge prism after it. This prism is used to refract back the correlation signal onto the optical axis. The angle of the reference beam used in making the hologram has been set equal to the refraction angle $\left(10^{\circ}\right)$ of the optical wedge prism. The hologram and the prism are mounted on a precision rotator driven by a stepper motor. The correlation spots are detected by a vidicon camera and then digitized, displayed, and analyzed by a PDP11/40 minicomputer system.

Figure 3(a) shows the original diatom picture chosen for this experiment. Since the interior structure is different for each diatom only the outline (b) is traced out and used in making the hologram. ${ }^{8}$ In Fig. $3(\mathrm{c})$ is 
the central portion of the MSF made from the object (b). Figure 4 shows a processed MSF plate in its holder (left), which consists of sixteen holograms of various ratios of the signal-to-reference beam intensity and exposure time. The best filter producing the maximum autocorrelation signal is cut out and glued on the disk (middle). Then the disk is held in the positioner (right) with three thin nylon screws, which center the filter on the axis of rotation. The heart of the rotational MSF

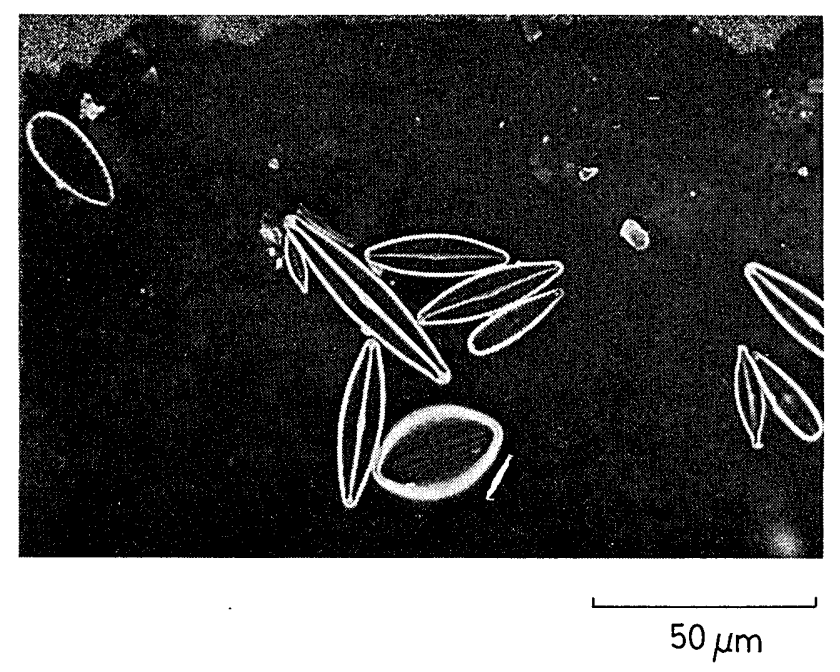

Fig. 1. Typical input pattern of diatoms taken through microscope. Scale indicates actual size of diatoms.



(a)

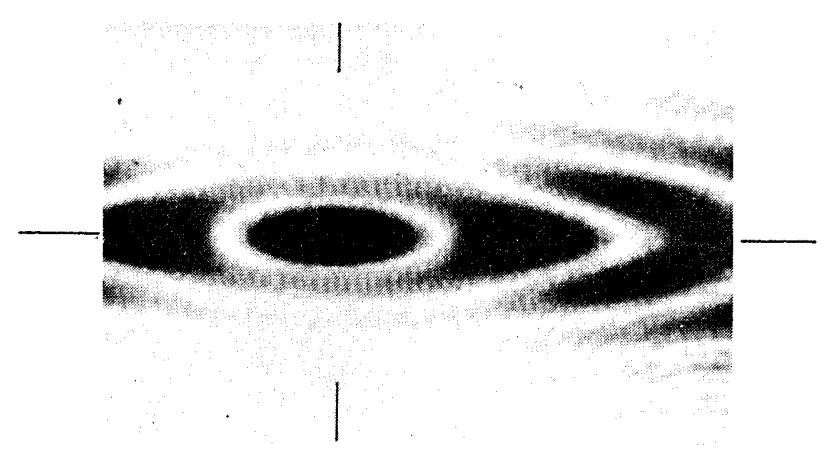

(c)

Fig. 3. (a) Original pattern of diatom under test. (b) Outline traced from (a) and used to make MSF (c). Only a portion of MSF is shown. system is seen in Fig. 5. The rotator is supported by an $X-Y$ translation stage, which centers the axis of the rotator on the optical axis. The wedge prism is placed into the back side of the rotator after centering the filter on the axis of rotation. The minicomputer executes a program to scan the correlation spots with a TV digitizer (Hamamatsu C-1000), analyzes the data, drives the stepper motor, and reads the angle of the filter with an encoder.

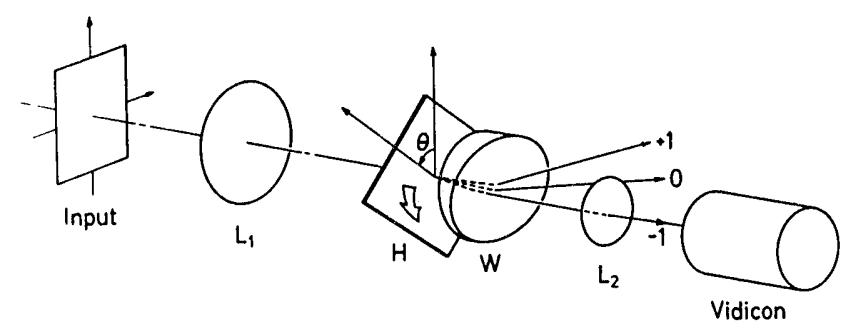

Fig. 2. Schematic illustration of rotational MSF. Optical wedge prism $W$ is used to center correlation signal on detector element of the vidicon.

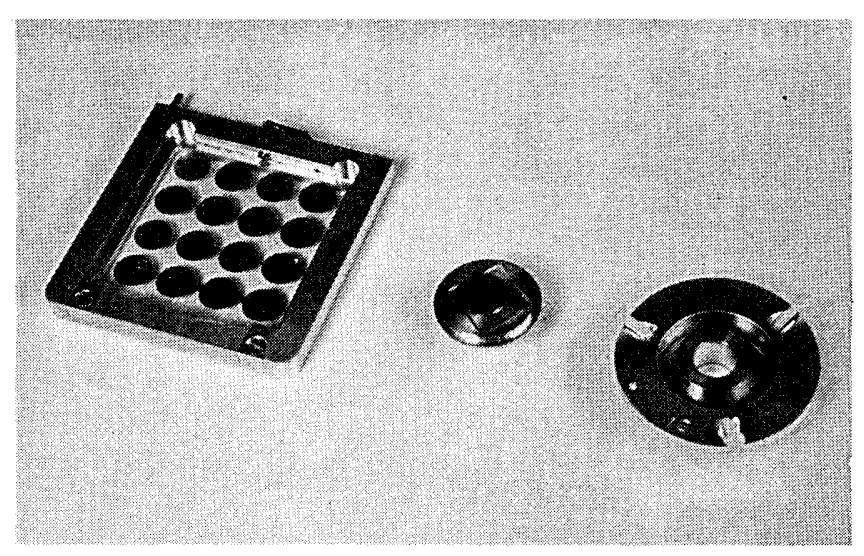

Fig. 4. MSF mounted on holder for recording (left) and rotation.

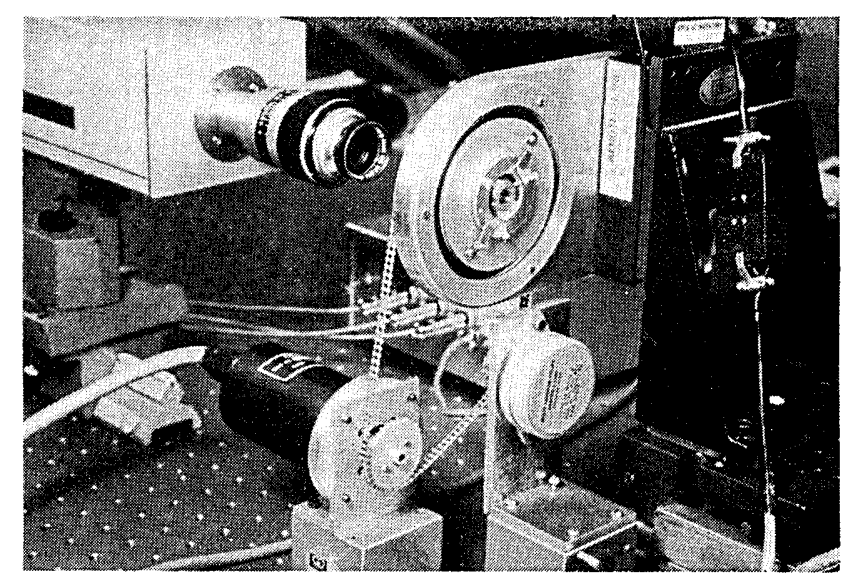

Fig. 5. Rotational MSF with stepper motor, encoder readout system, and TV camera. 


\section{Results and Discussion}

We first analyzed the changes of the correlation spot according to the rotation of the filter. Figures 6(a)-(e) show the variations of the spots produced by the pattern in Fig. 3(b) correlating with itself but at different angles. The two spots are shown in (a)-(c) coming closer to each other, finally overlapping at the same orientation in (d), and then separating again in (e). The intensity distributions of these patterns are digitized and plotted out three dimensionally. Figures 7(a)-(e) show the central portion of the correlation signals with $100 \times 100$ pixels and 256 gray levels. The maximum height is seen at (a) $\theta=0^{\circ}$, which drops by half at $\theta= \pm 1^{\circ}$ and then gradually spreads out into double peaks with similar heights. Now we see several problems involved in rotational filtering. The first is that one diatom can produce two spots with various distances and orientation. There is no longer a one-to-one correspondence between the number of diatoms and the correlation spots. This is true even for a diatom having the same shape as the original one stored in the hologram. We also noticed that the cross-correlation signal with different sized diatoms of the same species sometimes splits into two spots at $\theta=0^{\circ}$ or when the input and filter are in the same orientation. For example, the correlation with a larger diatom produces fairly bright double peaks, while the correlation with a smaller one produces weak double peaks. As is seen in Fig. 1, input patterns are usually a mixture of different sized diatoms. Hence, the number of correlation spots may not give any information on the number of diatoms to be identified. Actually, it is not easy to recognize even a single peak if it is spread out into several scanning lines or pixels on the vidicon. It takes a few minutes with our present system to analyze such data and recognize them as a single peak. Since the analysis must be repeated in at least 1-deg steps, this peak count may not be possible in practice.

To find a solution for these problems, we count the number of points, or pixels, over which the light intensity is above a particular threshold level. This process, which we call pixel counting, requires some simple software, or it can be carried out in hardware by passing the video signal through a comparator, converting it to a binary signal, and counting the number of binary pulses in one frame. Then we rotate the filter one step and repeat the process. The variations of the pixel counts as a function of the angle of the MSF now become important in finding diatoms distributed in random orientation.

The pixel counts also allow us to use an averaged filter made from various sized object patterns to cover wider ranges of size variation. Figure 8(a) shows the composite input of diatoms used in making the averaged filter. Sixteen different sized diatoms were selected randomly from a microscope slide, photographed, and printed in a larger scale. The outlines were traced from those pictures and distributed randomly with the same orientation. The composite input was then photo-

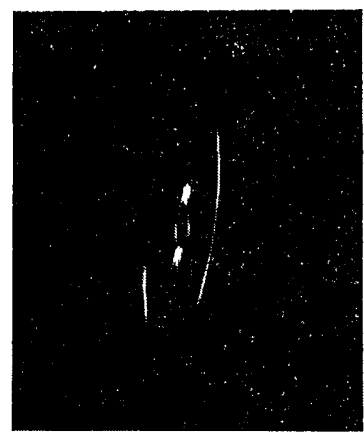

(a) $\theta=-8^{\circ}$

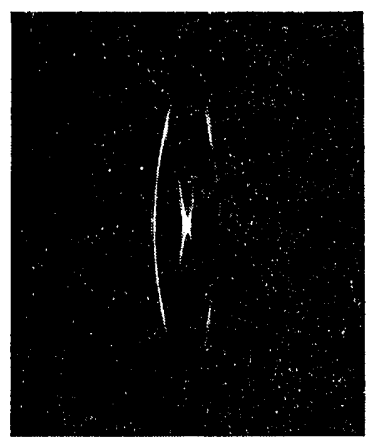

(d) $\theta=0^{\circ}$

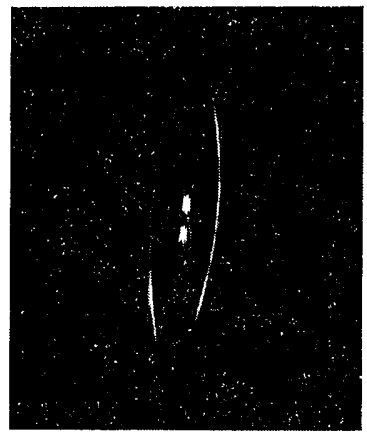

(b) $\theta=-4^{\circ}$

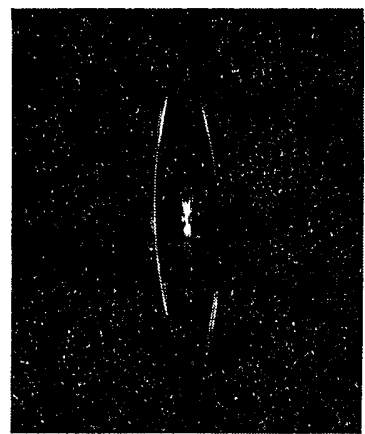

(e) $\theta=2^{\circ}$

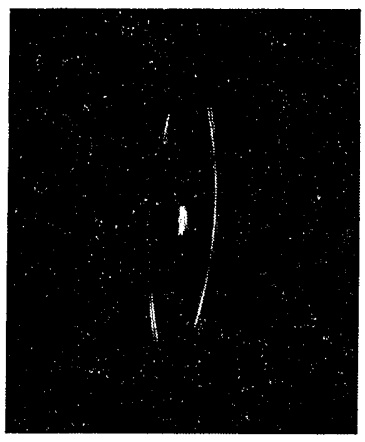

(c) $\theta=-2^{\circ}$

Fig. 6. Variations of correlation spots for object in Fig. 3(b) at different angles. 


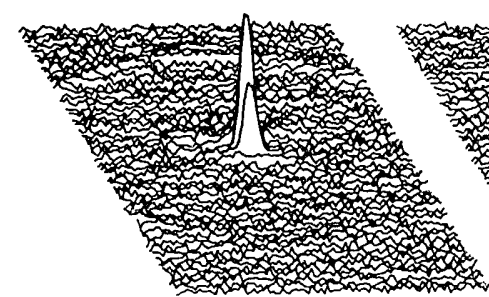

(a) $\theta=0^{\circ}$

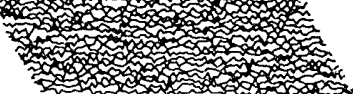

(b) $\theta=1^{\circ}$

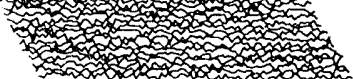

(c) $\theta=2^{\circ}$

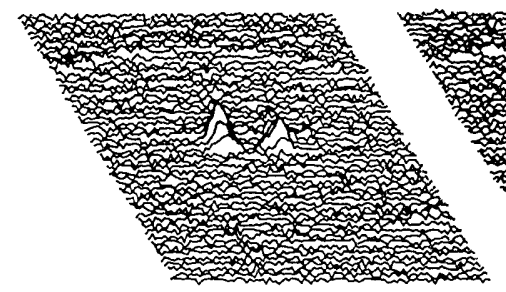

(d) $\theta=4^{\circ}$


2.0.5.

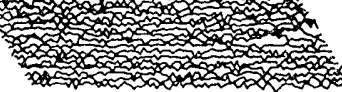

(e) $\theta=8^{\circ}$

Fig. 7. Three-dimensional plots of correlation peaks at different angles. These plots show $100 \times 100$ pixels quantized to 256 gray levels.

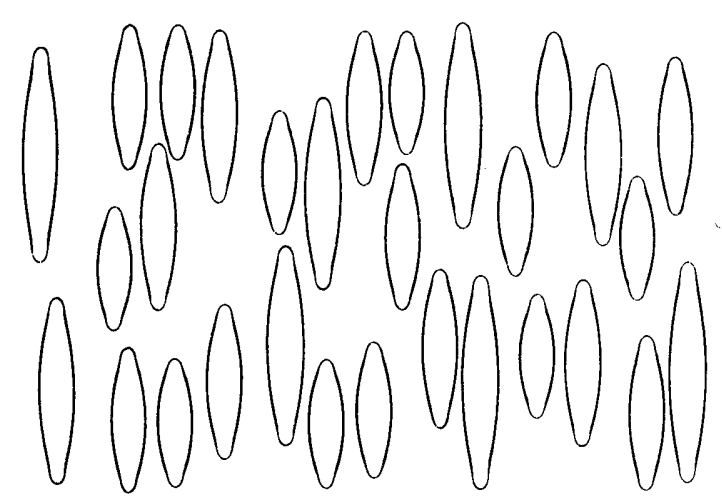

(a)

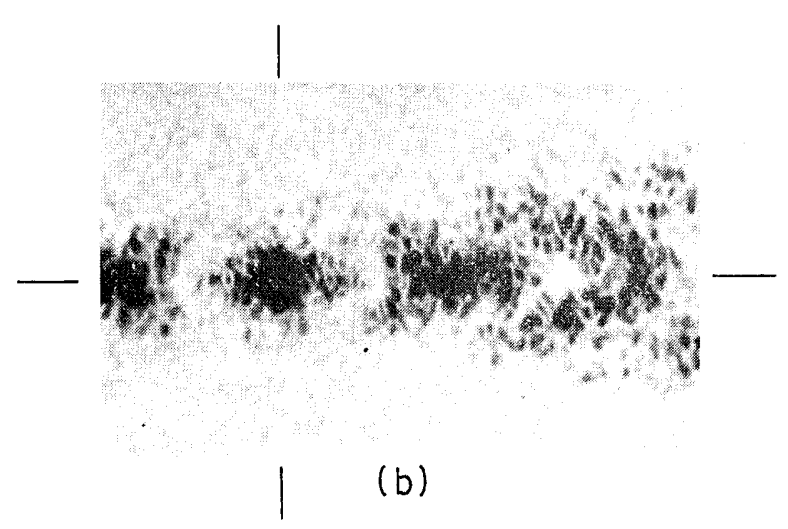

(a)
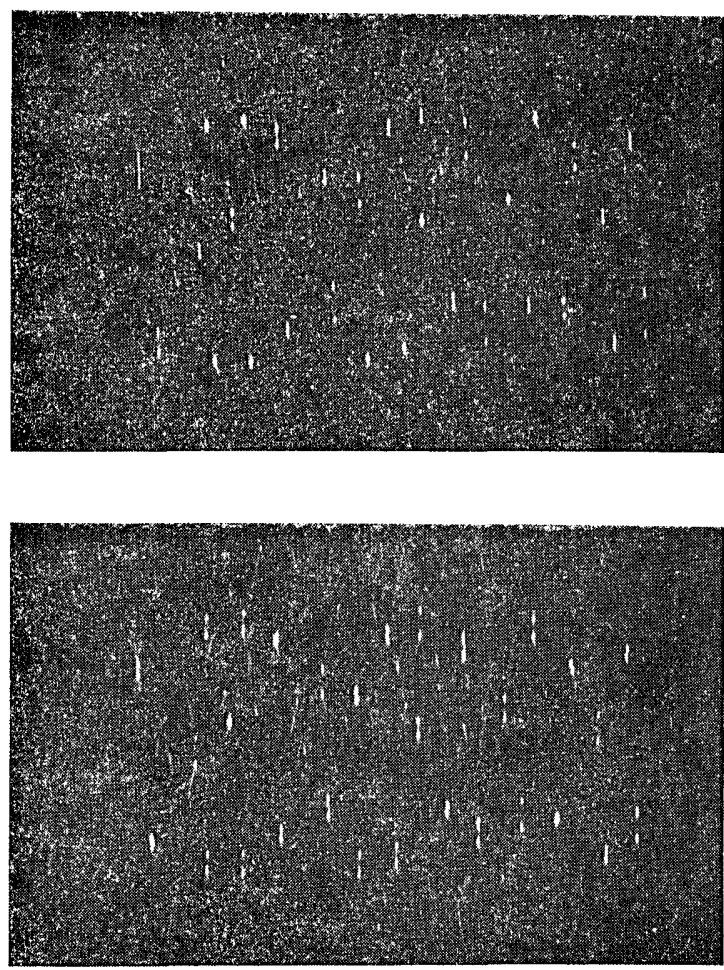

(b)
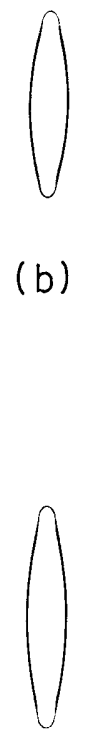

(c)

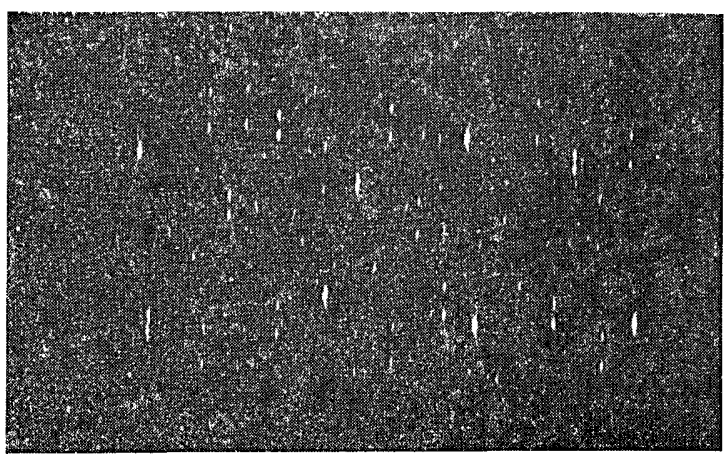

Fig. 8. Composite input (a) used in making the averaged MSF (b). 


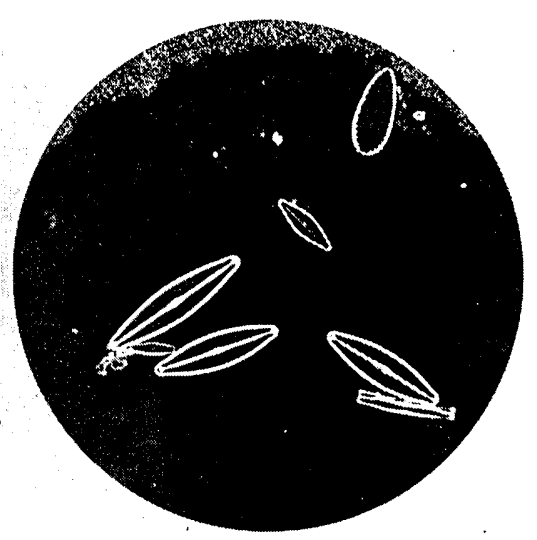

(a)

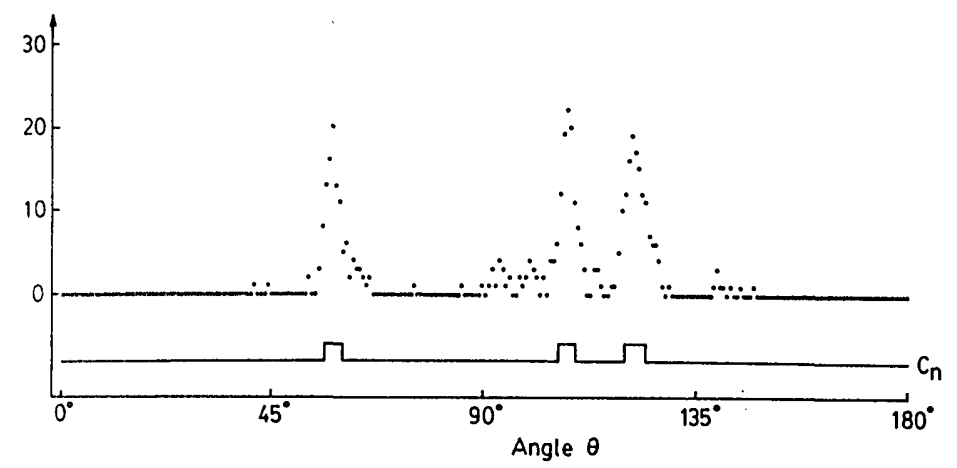

(c)

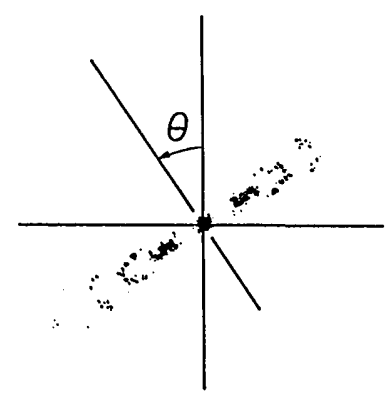

(b)

Fig. 10. (a) Actual photomicrographic picture to be analyzed and (c) results of pixel count as function of angle of MSF illustrated in (b).

graphed (Kodalith Ortho film, type 3, Kodak) carefully to assure that the scale is the same as the original photomicrographic picture. In Fig. 8(b), the central portion of the averaged hologram made from the pattern in Fig. 8(a) can be seen. In contrast with Fig. 3(c), the hologram becomes granular, and only the central spot, i.e., the dc component of the input, is seen to be highly saturated. ${ }^{13}$ Each grain consists of the fine holographic fringes, which diffract the correlation signal onto the output plane producing many spots.

In Fig. 8(a), the number of smaller sized diatoms was purposely increased as compared with the larger ones. The effect is seen in Figs. 9(a)-(c). These three photographs show the correlation spots of the composite pattern [Fig. 8(a)] against three different sized diatom patterns. Some brighter spots are seen at various points where similar sized objects were located in the original composite input. It is also noticed that the larger diatom in Fig. 9(c) produces larger and brighter spots due to its greater total transmission of light. Then, the number of pixels above a threshold level becomes generally greater for a larger object. As we mentioned, we put more smaller than larger diatoms in the composite to compensate for this factor. It is seen, therefore, that the smaller diatom produces many smaller correlation spots [Fig. 9(a)], while the larger one shows a few larger spots as in Fig. 9(c). The number of pixels above a threshold level was almost the same in these three correlation patterns. Diatoms of interest in a certain orientation are thus recognized by detecting the correlation patterns, as in Figs. 9(a)-(c), appearing on the TV screen as the filter rotates. The pixel count was done every $0.72 \mathrm{deg}$ of the filter angle $\theta$ for various photomicrographic input pictures. Figure 10(a) is an example of the input pattern $(\times 400$, High-Contrast Copy film, type 5069, Kodak), and (b) shows the orientation of the filter against in input (a). In Fig. 10(c) the result of the pixel count is plotted against the rotation angle $\theta$. The threshold level was chosen to be 140 , which gives us the maximum counts for diatoms to be identified but the minimum counts for all other objects. In Fig. 10(a), there are three diatoms to be identified. At around $\theta=60^{\circ}$, the diatom in the lower right part of the picture produces a maximum pixel count, as in Fig. 10(c). Further rotation of the filter produces two peaks with similar heights at corresponding angles for the two different sized diatoms at the lower left of Fig. 10(a). These signals are repeated in the next half turn (180$360^{\circ}$ ) of the filter because of the symmetry of this species. The number of peaks $C n=3$ in the variation of the pixel count is stored in memory and can be accessed later to tell us the number of diatoms of interest 




(a)

Fig. 11. Similar results as in Fig. 10 for different input pattern.

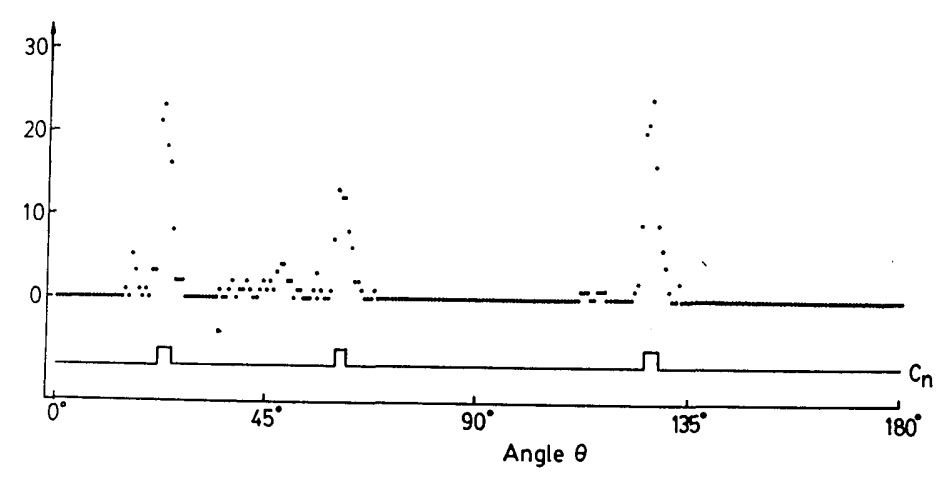

(b) in a given input pattern. Figures 11(a) and (b) are similar results for another input pattern.

In summary, the technique of rotational MSFs seems to be useful in biological pattern recognition. The pixel count not only minimizes the time of signal analysis but allows us to introduce averaged filtering techniques. Replacing the vidicon camera by a photodiode matrix array provides a more rapid analysis by increasing data throughput the system.

The present technique may also be useful in finding angular distributions of objects or detecting disorderliness and misalignment of objects.

The authors wish to thank Judy K. Partin for helpful discussions and Robert W. Ross, without whose precision tooling on the rotator the present experiment would not have been possible. This research was supported by the National Science Foundation (ASRA division under grant ENV-7710184).

\section{References}

1. A. Vander Lugt, F. B. Rotz, and A. Klooster, Jr., in Optical and Electro-Optical Information Processing, J. T. Tippett et al., Eds. (MIT Press, Cambridge, Mass., 1965), p. 125.

2. R. Herold and Kenneth Leib, Grummann Aerospace Corp., Bethpage, N. Y., RM-627 (Jan. 1977).

3. J. Cairns, Jr., K. L. Dickson, J. P. Slocomb, S. P. Almeida, J. K. T. Eu, C. Y. C. Liu, and H. F. Smith, in Trace Substances in Environmental Health-8, D. Hemphill, Ed. (U. Missouri Press, Columbia, 1974), p. 223.

4. R. Patrick, M. H. Hohn, and J. H. Wallace, Proc. Natl. Acad. Sci. U.S.A. 259, 12 (1954).

5. R. L. Kaesler and J. Cairns, Jr., Am. Midl. Nat. 88, 56 (1972).

6. J. Cairns, Jr., K. L. Dickson, G. R. Lanza, S. P. Almeida, and D. D. Balzo, Arch. Microbiol. 83, 141 (1972).

7. S. P. Almeida and J. K. T. Eu, Appl. Opt. 15, 510 (1976).

8. H. Fujii and S. P. Almeida, Appl. Opt. 18, 1659 (1979).

9. B. M. Watrasiewicz, Opt. Laser Technol. 4, 288 (1972).

10. D. W. Swift, Opt. Laser Technol. 4, 174 (1972).

11. C. J. Vienot, P. Smigielske, and H. Roger, Holographique Optique Developments Application (Dunod, Paris, 1971).

12. A. Vander Lugt, Appl. Opt. 5, 1760 (1966):

13. S. P. Almeida and H. Fujii, Appl. Opt. 18, 1663 (1979). 